\title{
Brief Report: Impact of COVID-19 in Individuals with Autism Spectrum Disorders: Analysis of a National Private Claims Insurance Database
}

\author{
Arun Karpur $^{1}$ (D) Vijay Vasudevan $^{1} \cdot$ Andy Shih $^{1} \cdot$ Thomas Frazier $^{1}$ \\ Accepted: 18 May 2021 / Published online: 26 May 2021 \\ (c) The Author(s), under exclusive licence to Springer Science+Business Media, LLC, part of Springer Nature 2021
}

\begin{abstract}
The COVID-19 pandemic continues to have a detrimental impact on individuals with disabilities. Data from FAIR Health's FH® NPIC (National Private Insurance Claims) database, one of the nation's largest databases of private insurance claim records, were analyzed to understand the experiences of individuals with ASD in the COVID-19 pandemic. Multivariate logistic regression models revealed that individuals with ASD+ID were nine times more likely to be hospitalized following COVID-19 infection (OR =9.3; 95\% CI: 6.9-12.5) and were nearly six times more likely to have an elevated length of hospital stay $(\mathrm{OR}=5.9 ; 95 \% \mathrm{CI}: 3.5-10.1)$ compared to those without ASD + ID. These findings point to the need for prioritizing access to vaccines to prevent COVID-19 infection and morbidities. This is the first study to illustrate a higher likelihood of hospitalization and elevated length of hospital stay from COVID-19 in individuals with ASD and other comorbidities.
\end{abstract}

Keywords Autism spectrum disorders · COVID-19 pandemic $\cdot$ Hospitalization $\cdot$ Intellectual disabilities $\cdot$ FAIR health inc.

\section{Introduction}

The global pandemic from SARS-CoV-2 virus infection causing the Coronavirus disease in 2019 (COVID-19) has led to wide-ranging crises in health and social supports for vulnerable individuals, especially for those with developmental disabilities and autism spectrum disorders (ASD). A study of individuals with ASD in Germany illustrated that the COVID-19 pandemic increased ASD-related behavioral difficulties, reduced sleep quality, and increased

Research for this article is based upon healthcare claims data compiled and maintained by FAIR Health, Inc. The authors are solely responsible for the conclusions reflected in this article. FAIR Health, Inc. is not responsible for the any of the opinions expressed in this article.

Due to uncertainty in diagnostic codes in the earlier months of the pandemic (February 2020-May 2020), any hospitalization reported within the three days prior to fifteen days after the date of the COVID-19 diagnostic code assumes the patient was admitted due to COVID-19 symptoms or complications. Hospital claims data in the later months had specific code of COVID-19. This way we captured the most relevant information.

Arun Karpur

arun.karpur@autismspeaks.org

1 Autism Speaks, 1060 State Rd, Princeton, NJ, USA hypersensitivity (Mutluer et al., 2020). People with ASD also had difficulty understanding the need for social distancing and staying at home, consistently using personal protective equipment (e.g., masks, gloves, etc.). Further, their caregivers did not have access to resources to educate their child with ASD about COVID-19 and ways to prevent infections. Finally, the study illustrated that increases in ASD-related behavioral challenges were correlated with the severity of anxiety in parents and caregivers. Similarly, another study from Northern Italy showed an exacerbation of pre-existing behavioral challenges in individuals with ASD, and parent-reported challenges in managing free time and structured activities during the COVID-19 pandemic (Colizzi et al., 2020). Children with ASD that did not receive services from schools experienced more intense behavioral problems.

Beyond the direct impact on the behavior and physical health of individuals with ASD, the COVID-19 pandemic surfaced several related challenges, such as food insecurity and mental health problems among households (Jeste et al., 2020). As educational and public health institutions grapple with optimal protocols for school openings in the US, the data on clinical experiences, including the prevalence of COVID-19 and hospitalization during the COVID-19 pandemic for individuals with ASD are weak to nonexistent. It is postulated that since individuals with ASD have a higher 
concentration of proinflammatory cytokines (Jyonouchi, 2013; Jyonouchi, Geng, Rose, Bennuri, \& Frye, 2019), seen in other chronic conditions that predispose individuals to COVID-19, the prevalence of COVID-19 infection is likely higher. The overlap in neuroinflammatory pathophysiology in ASD and COVID-19, is likely to exacerbate challenging behaviors and mental health problems (Lima et al., 2020). A recent case series of sixteen patients with ASD and COVID19 disease indicated that while most hospitalized individuals with ASD exhibited common symptoms of the infection, several had idiosyncratic manifestations contributing to challenges in diagnosis and treatment (Nollace et al., 2020).

In an analysis utilizing the TriNetX COVID-19 Research Network Data, consisting of electronic medical records from 42 health systems in the US, Turk et al. illustrated that the case fatality rates for individuals with intellectual and developmental disabilities (IDD) was comparable to the general population. However, they also identified an age-related trend where the case fatality was higher among younger individuals with IDD than those without IDD, which is likely due to a higher proportion of comorbidities among young individuals with IDD (Turk et al., 2020). While it was not reported, the study sample likely included many individuals with ASD grouped under the broad definition of IDD, as nearly $40 \%$ of individuals with ASD have a cooccurring ID (Maenner, Shaw, \& Baio et al., 2020). To our knowledge, no prior study has examined the experiences of hospitalization, including the duration of hospital stay among individuals with ASD in the COVID-19 pandemic. Thus, the purpose of this analysis is to illustrate the impact of COVID-19 infection on the health of individuals with ASD when compared to their peers with other chronic conditions.

\section{Methods}

\section{Data Source}

Claims records from February 1, 2020 through September 30, 2020 from the FAIR Health National Private Insurance Claims $\left(\mathrm{FH} \mathrm{NPIC}^{\circ}\right.$ ) database were utilized for this analysis. The Centers for Medicare and Medicaid Services (CMS) Chronic Conditions Data Warehouse (CCW) definitions were applied to the diagnostic codes attached to the claims data. Additionally, diagnostic codes for each claim were examined retrospectively until October 1, 2017, to allow for capturing diagnosis of ASD and other listed CCW conditions from prior visits (for more information, see: https:// www2.ccwdata.org/web/guest/condition-categories). Professional and facility claims were examined to understand the prevalence of COVID-19 infection. Specifically, both U07.1, 2019-nCoV acute respiratory disease (established to be used starting on March 18, 2020); and B97.29, other coronaviruses as the cause of diseases classified elsewhere (the de facto code recommended for use to diagnose patients until the new code was available) were used to identify claims pertaining to COVID-19 infection the database. Due to uncertainty in diagnostic codes in the earlier months of the pandemic (February 2020-May 2020), any hospitalization reported within the 3 days before to fifteen days after the COVID-19 diagnostic code assumes the patient was admitted due to COVID-19 symptoms or complications. Hospital claims data in the later months had a specific code of COVID-19.

A cohort of $35,898,076$ patients who incurred a claim between February 1, 2020, and September 30, 2020, were analyzed. The following condition groups were created based on the diagnostic codes as dummy variables $(1=$ has this condition; $0=$ does not have this condition):

Autism Spectrum Disorders (ASD only); Autism Spectrum Disorders AND Intellectual Disabilities and Related Conditions $(A S D+I D)$; Autism Spectrum Disorders AND Other Developmental Delays $(A S D+D D)$; Intellectual Disabilities and Related Conditions; Attention-deficit/Hyperactivity disorder(ADHD), Conduct Disorders, and Hyperkinetic Syndrome; Learning Disabilities; Other Developmental Delays; Hypertension; Chronic Kidney Disease; Hyperlipidemia; Anemia; Diabetes; Ischemic Heart Disease; Heart Failure; Obesity; Rheumatoid Arthritis/Osteoarthritis; Chronic Obstructive Pulmonary Disease; Atrial Fibrillation; Depressive Disorders; Anxiety Disorders; None of the Specified Conditions.

Specifically, claims for individuals with birth-related disorders (e.g., ASD, ID, DD) were assigned to their respective groups based on birth-related disorders to create mutually exclusive claim records. Claims that were likely to be adultonset chronic conditions were grouped into their respective diagnostic category without being mutually exclusive as it was difficult to ascertain their primary diagnosis given the length of clinical history claims available for the analysis.

\section{Statistical Analyses}

The descriptive analysis studied distribution in trends in hospitalization in the reported claims. Elevated length of stay was a categorical variable-longer than the median length of stay for all claims and shorter than the median length of stay. Two separate stepwise multiple logistic regression models were created to study the likelihood of hospitalization and experiencing elevated length of hospital stay. Each of the condition groups (e.g., ASD + ID, ASD + DD, ASD, etc.), coded as dummy variables, were entered along with gender (male; female) and age-groups (0-18 years; $19-29$ years; 30-39 years; 40-49 years; $50-59$ years; $60-69$ years; 70 years and older) using the stepwise forward logistic regression with a significance level of 0.3 required to allow 
the variable into the model and a significance level of 0.35 required to allow the variable to stay into the model with each subsequent entry. Stepwise regression models were used due to the exploratory nature of the analysis.

\section{Results}

Based on the analytical dataset, the overall prevalence of COVID-19 infection was estimated to be $2.04 \%$. Out of the total 144,147 individuals with ASD identified in the FAIR Health database who incurred a claim between February 2020 and September 2020, 1300 individuals had a positive COVID-19 diagnosis resulting in an overall prevalence of $0.90 \%$. A greater proportion of individuals with ASD + ID, ASD + DD, and ASD only were males and belonged to younger age groups (i.e., 0-18 years, and 19-29 years) compared to those in other condition groups. The estimated prevalence of COVID-19 infection was $2.01 \%$ among individuals with ASD + ID (201 cases out of 9991 individuals), $0.75 \%$ among individuals with ASD + DD (538 cases out of 71,999 individuals), and $0.90 \%$ among individuals with ASD only (561 cases out of 62,165 individuals). The highest prevalence of COVID-19 was among individuals with heart failure without ASD or other developmental conditions $(4.30 \%)$.

The overall mortality from COVID-19 for individuals in the analytical data set was $0.89 \%$. The proportion of deaths or mortality from COVID-19 in infected individuals was $2.49 \%$ among individuals with ASD + ID, $0.37 \%$ among individuals with ASD + DD, and $0.89 \%$ for individuals with ASD only. Individuals with heart failure without ASD or other developmental conditions had the highest proportion of mortality from COVID-19 (10.5\%). Table 1 illustrates the proportion of prevalence and mortality for the condition groups. The unadjusted data (Fig. 1) indicate larger proportions of outpatient doctor's office visits for individuals with ASD + ID, ASD + DD, and ASD only $(69.2 \%, 80.3 \%$, and $85.9 \%$ respectively). Further, as the proportion utilizing doctor's office declined, the proportion of claims with hospitalization increased.

The overall proportion of hospitalization for individuals with COVID-19 infection in the analytical data set was $12.70 \%$. Figure 2 illustrates the odds ratios for hospitalization for each of the condition groups based on the stepwise forward logistic regression model. After adjusting for age group and gender, individuals with ASD + ID were more than nine times likely to be hospitalized compared with those that did not have this condition $(\mathrm{OR}=9.3 ; 95 \%$
Table 1 Prevalence of COVID19 infection and mortality in condition groups

\begin{tabular}{llc}
\hline Condition groups & $\begin{array}{l}\text { Prevalence propor- } \\
\text { tion }(\%)\end{array}$ & $\begin{array}{l}\text { Mortality } \\
\text { proportion } \\
(\%)\end{array}$ \\
\hline Autism spectrum disorders and other developmental delays & & 0.37 \\
Learning disabilities & 0.75 & 1.21 \\
Autism spectrum disorders & 0.85 & 0.89 \\
Other developmental delays & 0.90 & 0.57 \\
None of the specified conditions & 0.95 & 0.02 \\
ADHD, conduct disorders, and hyperkinetic syndrome & 1.57 & 0.48 \\
Autism spectrum disorders and intellectual disabilities and related & 1.94 & 2.49 \\
conditions & & \\
Intellectual disabilities and related conditions & 2.21 & 5.44 \\
Depressive disorders & 2.51 & 2.02 \\
Anxiety disorders & 2.70 & 1.45 \\
Rheumatoid arthritis/osteoarthritis & 2.76 & 2.45 \\
Hyperlipidemia & 2.92 & 2.00 \\
Hypertension & 2.98 & 2.77 \\
Atrial fibrillation & 3.19 & 8.87 \\
Ischemic heart disease & 3.35 & 6.49 \\
Obesity & 3.51 & 1.85 \\
Diabetes & 3.60 & 3.19 \\
Chronic obstructive pulmonary disease & 3.74 & 5.23 \\
Chronic kidney disease & 3.81 & 7.17 \\
Anemia & 4.11 & 3.47 \\
Heart failure & 4.30 & 0.50 \\
\hline & & \\
\hline
\end{tabular}

Data table is sorted in the descending order of the prevalence proportion 


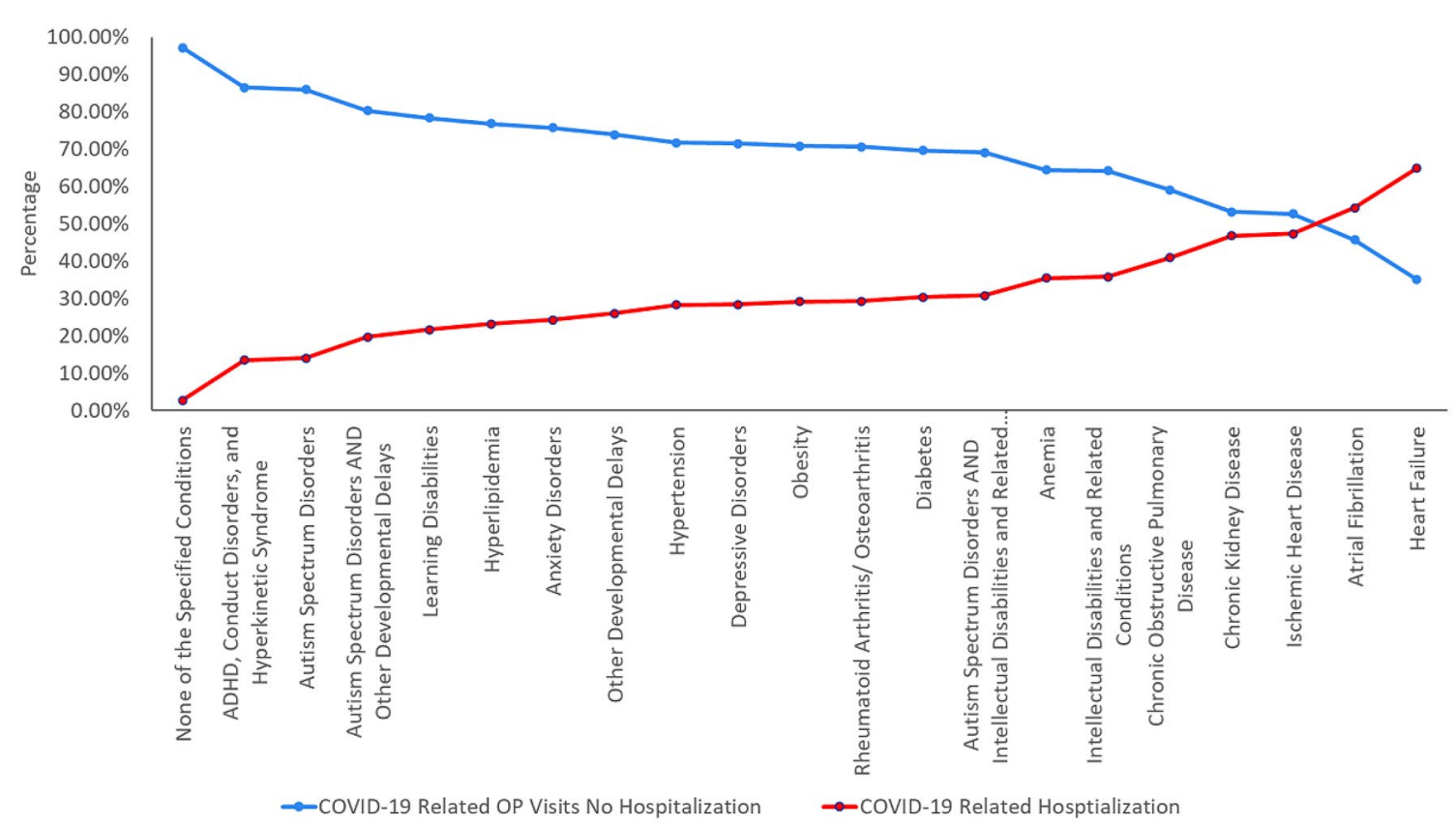

Fig. 1 Frequency of outpatient visits and hospitalizations in COVID-19 for condition groups

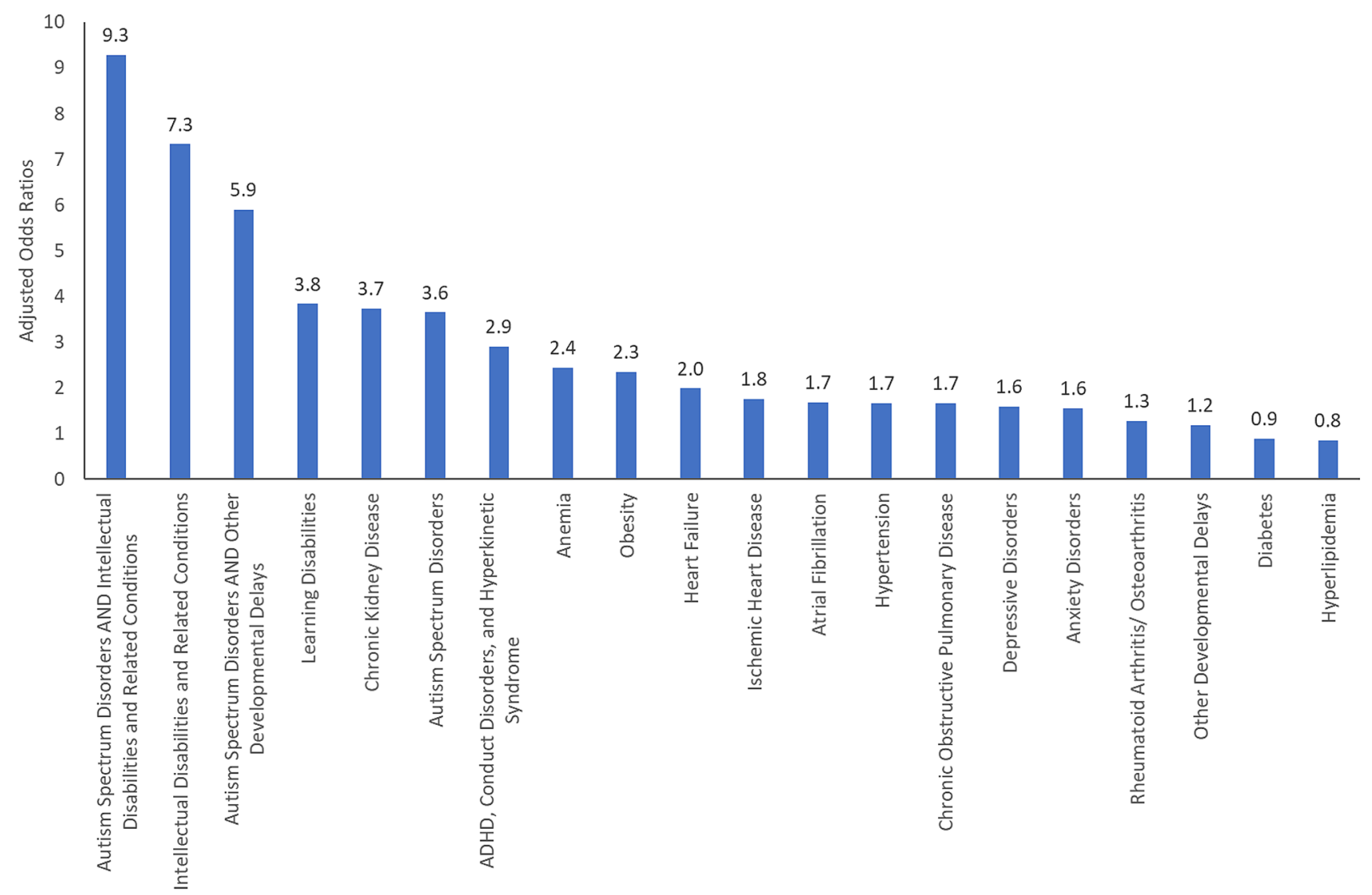

Fig. 2 Likelihood of hospitalization in COVID-19 infection across the condition groups 
CI: 6.9-12.5). Similarly, individuals with ASD + DD were nearly six times more likely (OR $=5.9$; 95\% CI: 4.7-7.3), and those with ASD only were almost four times more likely to be hospitalized (OR $=3.6$; 95\% CI: 2.9-4.6) compared to those that did not have this condition. The overall proportion of individuals with an elevated length of hospital stay (i.e., longer than median length) from COVID-19 infection was 5.58\%. Figure 3 illustrates the odds ratios for elevated length of hospital stay for each of the condition groups based on the stepwise forward logistic regression model. Individuals with ASD + ID were six times more likely to have elevated length of hospital stay than those who did not have this condition $(\mathrm{OR}=5.9$; 95\% CI: 3.5-10.1). Individuals with ASD + DD were four times more likely (OR $=4.0 ; 95 \% \mathrm{CI}: 2.7-5.9)$, and those with ASD only were nearly three times more likely to have elevated length of hospital stay $(\mathrm{OR}=2.8 ; 95 \% \mathrm{CI}$ : 1.8-4.5) compared to those that did not have this condition. Regression tables are provided in the supplementary materials.

\section{Discussion}

This is the first study to illustrate a higher likelihood of hospitalization and elevated length of hospital stay from COVID-19 infections for individuals with ASD and other comorbidities. While a lower proportion of individuals with ASD acquired COVID-19 infection than other condition groups, they were significantly more likely to be hospitalized for COVID-19 and have a longer length of stay in the hospital. Contrastingly, individuals with other chronic conditions had a higher prevalence of COVID19 infections (e.g., Heart Failure, Obesity, Diabetes) had lower adjusted odds of hospitalization and elevated length of hospital stay. Underlying demographic patterns likely drive a lower overall prevalence of COVID-19 in ASD. A closer examination of the demographic distribution indicates that more than $90 \%$ of individuals with ASD were in the younger age range (i.e., less than 30 years old). Nearly $70 \%$ belonged to the $0-18$-year-old group. As a result, most individuals with ASD in the data are likely living with their families during the pandemic, limiting their exposure compared to individuals in congregate living environments. A substantial increase in morbidity and

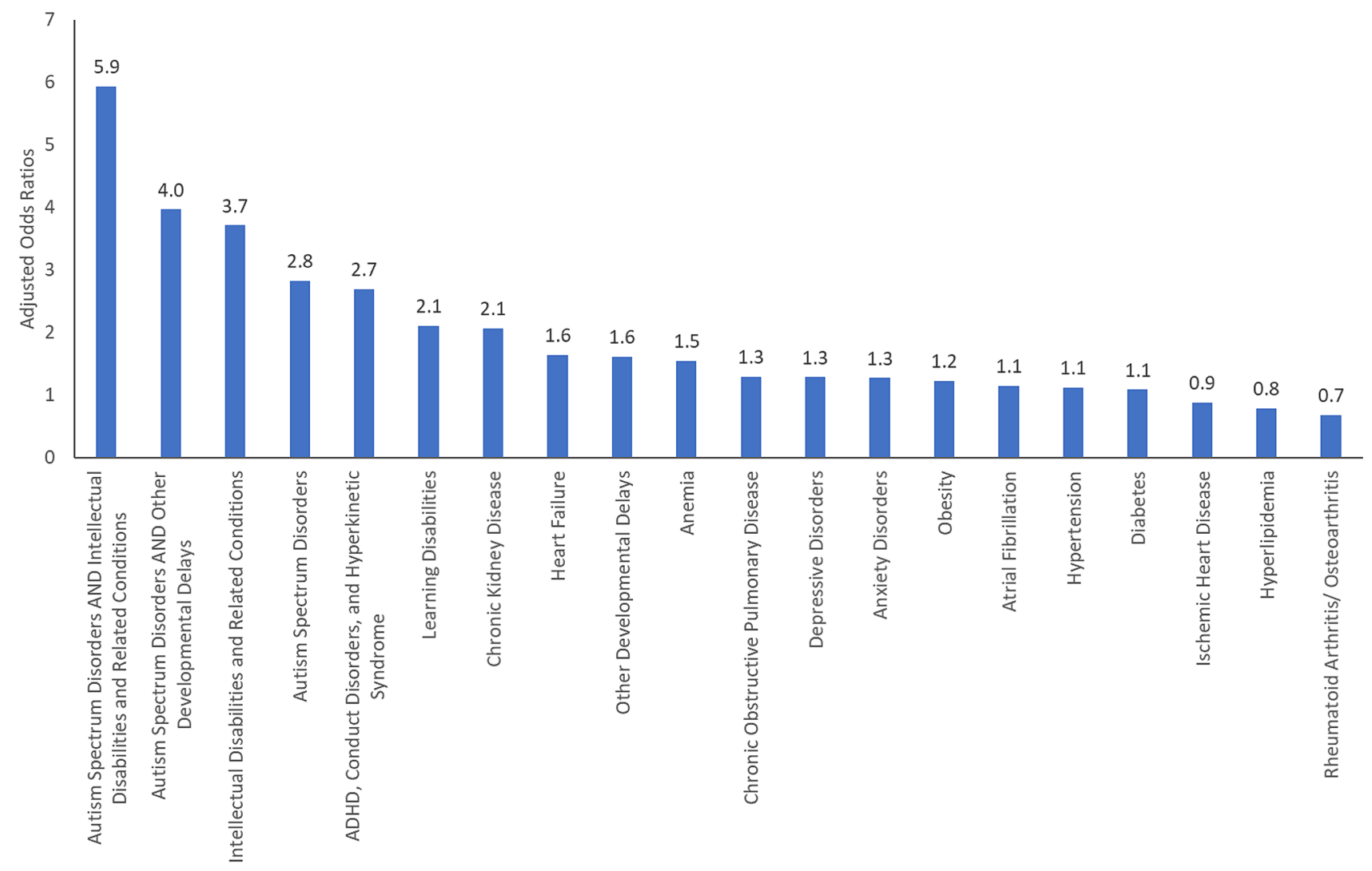

Fig. 3 Likelihood of higher than median length of stay in hospital from COVID-19 infection 
mortality in COVID-19 among individuals with disabilities, especially among individuals with intellectual and developmental disabilities, has been associated with their living situation in congregate care settings (Landes et al., 2020).

Once individuals with ASD acquire COVID-19 infection, the regression models indicate a higher likelihood of hospitalization and elevated length of hospital stay. While Cunningham et al. (2020) described a similar pattern of lower COVID-19 prevalence and higher morbidity among young individuals using electronic medical records data, the quantitative differences in the likelihood of hospitalization and duration of stay for individuals with ASD is concerning and requires attention (Cunningham et al., 2020). Individuals with ASD, in general, have higher medical needs resulting from cooccurring mental health and other conditions such as epilepsy, digestive disorders, etc. (Karpur et al., 2018; Shea et al., 2018). It is possible that the COVID-19 infection, irrespective of severity, precipitates behavior health challenges leading to hospitalization and longer duration of stay (Bal et al., 2021; Righi et al., 2017; White et al., 2021). Further, several immunological theories, including the more recent preposition of abnormal melatonin production among individuals with $\mathrm{ASD}$, might contribute to the increased severity of COVID-19 infection (Brown et al., 2021).

The findings of increased morbidity in COVID-19 are relevant from the perspective of growing discussion on the prioritization of populations for COVID-19 vaccines. Only a handful of states have considered prioritizing individuals with intellectual disabilities (see: https://www.kff.org/policy-watch/the-next-phase-of-vaccine-distribution-high-riskmedical-conditions/), and this does not include all individuals with ASD. However, given the challenges in consistently implementing social distancing practices and PPEs among individuals with ASD, it would be helpful to include them as one of the high-risk populations for immunizations.

A few factors limit these analyses. Firstly, the FH NPIC ${ }^{\odot}$ data are limited to private health claims data, while many people with ASD are on public insurance like Medicaid. Medicaid funds healthcare and long-term services and supports such as congregate or community-based housing for people with ASD or other developmental disabilities. Future studies should explore hospitalization and length of stay because of COVID-19 for people with ASD who have Medicaid. Secondly, the findings are based on claims data that trigger a record upon receipt of services, and the data capture events only through September 2020. The prevalence of COVID-19 infection may be much higher than is reported here in the analysis, and it indeed increased as infections increased in late fall/early winter. An additional limitation is that condition identification was based on the ICD-10 diagnostic codes that may miss some individuals. The claims data are also limited in attributing events of hospitalization resulting from key symptoms or complications of COVID-19.

Further, a substantial proportion of private claims data do not report on race/ethnicity, making it challenging to examine disparities in experiences ( $\mathrm{Ng}$ et al., 2017). Factors that indicate social and economic vulnerability, including living situation, would likely present more nuanced and policy-relevant findings. Future studies that employ natural language processing or medical record review to identify COVID-19, people with ASD, and other important factors related to hospitalization and length of stay would help extend the present work and further characterizing the impact of COVID-19 on the ASD population.

Supplementary Information The online version contains supplementary material available at https://doi.org/10.1007/s10803-021-05100-x.

Acknowledgments Authors also acknowledge contributions from FAIR Health Inc., specifically Ali Russo, Eric Okurowski, David Cheng, and Josh Smolinsky.

Author Contributions AK conceptualized the study, supervised data analysis, wrote the manuscript, TF conceptualized and contributed to the writing, VV contributed to the writing of manuscript, and AS helped with conceptualization of the study.

Funding No external funding was received for this research.

\section{References}

Bal, V. H., Wilkinson, E., White, L. C., Law, J. K., Feliciano, P., \& Chung, W. K. (2021). Early pandemic experiences of autistic adults: Predictors of psychological distress. Autism Research. https://doi.org/10.1002/aur.2480

Brown, G. M., Karthikeyan, R., Pandi-Perumal, S. R., \& Cardinali, D. P. (2021). Autism spectrum disorder patients may be susceptible to COVID-19 disease due to deficiency in melatonin. Medical Hypotheses. https://doi.org/10.1016/j.mehy.2021.110544

Colizzi, M., Sironi, E., Antonini, F., Ciceri, M. L., Bovo, C., \& Zoccante, L. (2020). Psychosocial and behavioral impact of COVID19 in autism spectrum disorder: An online parent survey. Brain Sciences. https://doi.org/10.3390/brainsci10060341

Cunningham, J. W., Vaduganathan, M., Claggett, B. L., Jering, K. S., Bhatt, A. S., Rosenthal, N., \& Solomon, S. D. (2020). Clinical outcomes in young US adults hospitalized with COVID-19. JAMA Internal Medicine. https://doi.org/10.1001/jamainternmed.2020. 5313

Jeste, S., Hyde, C., Distefano, C., Halladay, A., Ray, S., Porath, M., \& Thurm, A. (2020). Changes in access to educational and healthcare services for individuals with intellectual and developmental disabilities during COVID-19 restrictions. Journal of Intellectual Disability Research, 64(11), 825-833. https://doi.org/10.1111/jir. 12776

Jyonouchi, H. (2013). Immunological abnormalities in autism spectrum disorders. Advances in Neuroimmune Biology, 4, 141-159. https:// doi.org/10.3233/NIB-130061

Jyonouchi, H., Geng, L., Rose, S., Bennuri, S. C., \& Frye, R. E. (2019). Variations in mitochondrial respiration differ in IL-1B/IL-10 ratio based subgroups in autism spectrum disorders. Frontiers in Psychiatry. https://doi.org/10.3389/fpsyt.2019.00071 
Karpur, A., Lello, A., Frazier, T., Dixon, P. J., \& Shih, A. J. (2018). Health disparities among children with autism spectrum disorders: Analysis of the National Survey of Children's Health 2016. Journal of Autism and Developmental Disorders. https://doi.org/ 10.1007/s10803-018-3862-9

Landes, S. D., Turk, M. A., Formica, M. K., McDonald, K. E., \& Stevens, J. D. (2020). COVID-19 outcomes among people with intellectual and developmental disability living in residential group homes in New York State. Disability and Health Journal, 13(4), 100969-100969.

Lima, M. E. S., Barros, L. C. M., \& Aragao, G. F. (2020). Could autism spectrum disorders be a risk factor for COVID-19? Medical Hypotheses, 144, 109899. https://doi.org/10.1016/j.mehy.2020. 109899

Maenner, M., Shaw, K. A., Baio, J., Washington, A., Patrick, M., DiRienzo, M., Christensen, D., Wiggins, L. D., Pettygrove, S., Andrews, J. G., Lopez, M., Hudson, A., Baroud, T., Schwenk, Y., White, T., Rosenberg, C. R., Lee, L.-C., Harrington, R. A., Huston, M., ... Dietz, P. M. (2020). Prevalence of autism spectrum disorders among children aged 8 years-autism and developmental disabilities monitoring network, 11 sites, United States, 2016. Morbidity and Mortality Weekly Report, 69(4), 1-12.

Mutluer, T., Doenyas, C., \& Aslan Genc, H. (2020). Behavioral implications of the Covid-19 process for autism spectrum disorder, and individuals' comprehension of and reactions to the pandemic conditions. Frontiers in Psychiatry, 11, 561882. https://doi.org/ 10.3389/fpsyt.2020.561882

Ng, J. H., Ye, F., Ward, L. M., Haffer, S. C., \& Scholle, S. H. (2017). Data on race, ethnicity, and language largely incomplete for managed care plan members. Health Affairs, 36(3), 548-552. https:// doi.org/10.1377/hlthaff.2016.1044
Nollace, L., Cravero, C., Abbou, A., Mazda-Walter, B., Bleibtreu, A., Pereirra, N., \& Giannitelli, M. (2020). Autism and COVID-19: A case series in a neurodevelopmental unit. Journal of Clinical Medicine. https://doi.org/10.3390/jcm9092937

Righi, G., Benevides, J., Mazefsky, C., Siegel, M., Sheinkopf, S., \& Morrow, E. (2017). Predictors of inpatient psychiatric hospitalization for children and adolescents with autism spectrum disorder. Journal of Autism and Developmental Disorders, 48(11), 3647-3657.

Shea, L. L., Xie, M., Turcotte, P., Marcus, S., Field, R., Newschaffer, C., \& Mandell, D. (2018). Brief report: Service use and associated expenditures among adolescents with autism spectrum disorder transitioning to adulthood. Journal of Autism and Developmental Disorders. https://doi.org/10.1007/s10803-018-3563-4

Turk, M. A., Landes, S. D., Formica, M. K., \& Goss, K. D. (2020). Intellectual and developmental disability and COVID-19 casefatality trends: TriNetX analysis. Disability and Health Journal, 13(3), 100942-100942.

White, L. C., Law, J. K., Daniels, A. M., Toroney, J., Vernoia, B., Xiao, S., \& Chung, W. K. (2021). Brief report: Impact of COVID-19 on individuals with ASD and their caregivers: A perspective from the SPARK cohort. Journal of Autism and Developmental Disorders. https://doi.org/10.1007/s10803-020-04816-6

Publisher's Note Springer Nature remains neutral with regard to jurisdictional claims in published maps and institutional affiliations. 